\title{
Mammary like gland adenoma with GATA-3 expression - a benign vulval tumor with mammary differentiation often misdiagnosed as adenocarcinoma
}

\author{
Deval Parekh, Sunaina Wadhwa, Asima Mukhopadhyay, Angad Singh \\ Corresponding author: Dr. Angad Singh, Department of Pathology, Tata Medical Center, Kolkata, \\ India; Email : drangad@gmail.com
}

Distributed under Attribution-Non Commercial - Share Alike 4.0 International (CC BY-NC-SA 4.0)

\section{ABSTRACT}

Papillary hidradenoma/ mammary-like gland adenoma (MLGA) is a benign glandular tumor of the vulva. It presents as a mass lesion which often undergoes secondary ulceration and bleeding resembling a malignant tumor. It arises from specialized anogenital glands in the labial folds which resemble mammary glands on microscopy. The tumour shows a tubulo-papillary architecture and is composed of bland columnar cells with apical snouting. GATA-3 is a transcription factor and it's expression is essential for normal mammary gland development. GATA-3 immunohistochemistry (IHC) is positive in luminal cells of breast and its positive in ductal hyperplasia as well as in breast carcinoma. In summary, we describe a case of a benign vulval mammary gland like adenoma with diffuse GATA-3 expression which was originally diagnosed as a malignant low-grade polymorphous adenocarcinoma and discuss its clinical and associated histopathological findings.

Keywords: Mammary like gland adenoma, papillary hidradenoma, vulva, GATA-3.

Mammary like glands (MLGs) are normal structures of the anogenital region. They are distributed in an elliptical fashion involving the preclitoral region, interlabial sulcus, fourchette, perineum and perianal region. ${ }^{1}$ These glands have morphological resemblance to mammary tissue and are distinct from eccrine and apocrine glands of anogenital region. The presence of these glands explains the development of breast like lesions in vulva viz. lactating mammary gland, fibroadenoma, papillary hidradenoma, extramammary paget's disease. Van der Putte et al first suggested MLGs as the site of origin for breast like tumors in vulva. $^{2}$

Currently, the WHO classification of tumors of the vulva incorporates the term 'Papillary Hidradenoma' for this tumor. Mammary like gland adenoma (MLGA) is considered as its synonym. We describe a case of a vulvar MLGA with nuclear expression of GATA-3 and discuss its significance.
Case

A 46 years old female patient was evaluated outside for a painless nodular swelling in vulva for 6 months. The swelling was gradually increasing in size. Clinical examination done outside showed a $2 \mathrm{~cm}$ solid, nodular thickening in the labia majora. The urethral and anal openings were uninvolved. Patient underwent a biopsy of the lesion. Histopathological examination of the specimen done outside suggested a diagnosis of a low grade polymorphous adenocarcinoma. Thereafter, the patient presented at our centre and local examination showed an ill-defined thickening of labia majora. We received H\&E stained slides and blocks for histopathological review at our centre.

Histopathology: Slides examined showed two bits of tissue. The larger bit showed a nodular rounded tumor arranged in atubulo-papillary and glandular architecture (figure 1). The glands and papillae were formed by

Received: $12^{\text {th }}$ May 2020, Peer review completed: $20^{\text {th }}$ October 2020, Accepted: $5^{\text {th }}$ January 2021.

Parekh D, Wadhwa S, Mukhopadhyay A, Singh A. Mammary like gland adenoma with GATA-3 expression - a benign vulval tumor with mammary differentiation often misdiagnosed as adenocarcinoma. The New Indian Journal of OBGYN. 2021; 8(1): 143-45. 


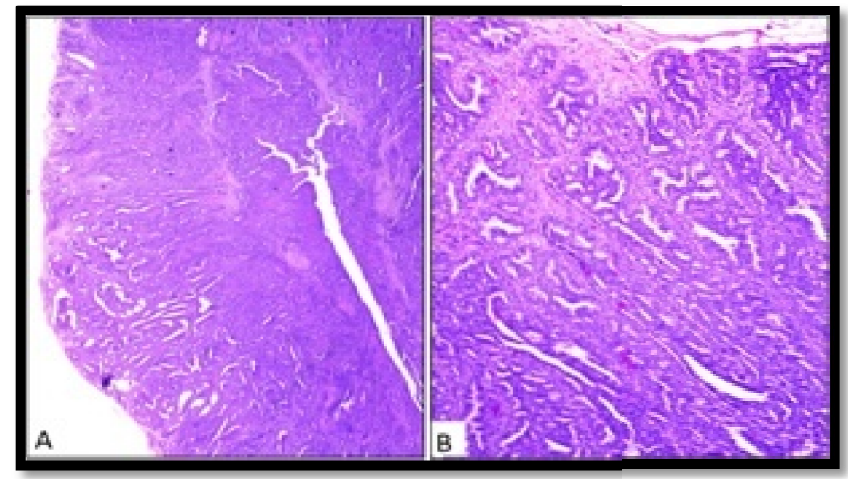

Figure 1: (A) HE low power (2x) view of tumor showing a circumscribed nodular outline; (B) HE (10x) shows the tumor in a glandular and papillary architecture.

monomorphic columnar cells with minimal atypia. Nuclei were small, round with fine granular chromatin and inconspicuous nucleoli. Focal apical cytoplasmic snouts were also seen in few cells. No significant nuclear atypia or mitotic activity was appreciated. At the periphery of these glands, some flattened cells with elongated short spindled nuclei were also seen, however they were largely inconspicuous on H\&E examination. The separate smaller bit of tissue showed vulvar tissue with lining squamous mucosa and presence of focal mammary like glands in subepithelial tissue. These glands showed an irregularly dilated duct like structure at centre which was lined by cuboidal to low columnar epithelium along with surrounding stromal tissue. Immunohisto-chemistry (IHC) for p63 and S100 highlighted the lining myoepithelial cells around each gland (figure 2). The glandular epithelial cells were diffusely positive for ER, GATA-3 and focally positive for GCDFP-15 (figure 2). IHC for PAX-8 and p16 was negative. The Ki-67 proliferation index was $5-6 \%$. A diagnosis of mammary-like gland adenoma was confirmed.

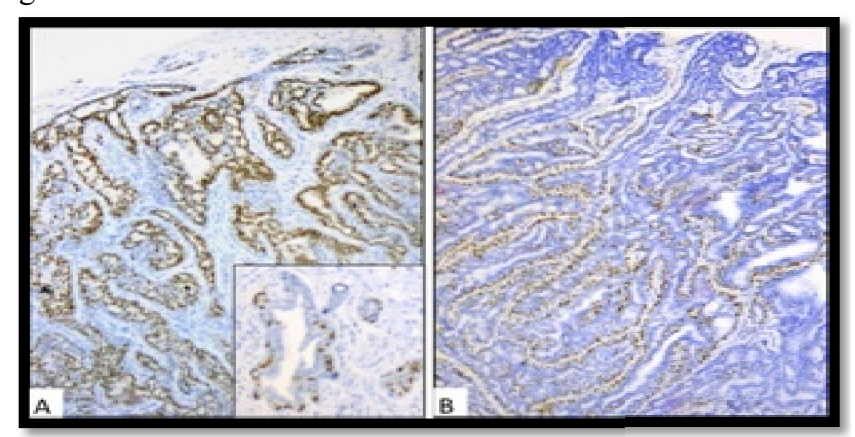

Figure 2: (A) GATA-3 IHC (10x) shows diffuse nuclear positivity, inset shows focal GATA-3 staining in a normal mammary like gland in adjacent tissue; (B) IHC for p63 (10x) highlights myoepithelial cells lining glands $\&$ papillae.
Course: A wide local excision of thickened part of labia majora was done in which $2.5 \mathrm{~cm}$ area was excised. Histopathological examination showed vulval tissue with normal anogenital mammary like glands in subepithelium. No residual tumor was seen. Patient had an uneventful recovery and on follow up for 12 months, has no recurrence.

\section{Discussion}

GATA family of transcription factors play an important role in activation or repression of multiple target genes. It helps in recruitment of chromatin remodelling complexes and is expressed in a tissue specific manner. ${ }^{3}$ GATA-3 has a critical role in gene regulation in T cells, mammary glands and the central nervous system. GATA-3 expression is seen in all stages of mammary development, but is restricted to the luminal cells while abluminal (myoepithelial) cells are negative. It's expression can be assessed using IHC. GATA3 IHC is widely used in the setting of a metastatic carcinoma to confirm origin from a suspected breast or urothelial primary. Nuclear positivity is seen in approximately $50 \%$ of normal breast tissue, $78 \%$ of ductal hyperplasia and $90 \%$ of ductal carcinoma of breast. In our case, GATA-3IHC showed strong nuclear positivity in the tumor as well as adjacent mammary type anogenital glands. The WHO classification of vulval tumors describes these specialized anogenital glands to be the origin of papillary hidradenoma/MLGA. In our view, the term mammary like gland adenoma is a more accurate description of this entity as it morphologically and immunohistochemically resembles an adenoma of the breast.

MLGA physically appears as a red, pink or skin coloured solid lesion. Few cases may be ulcerated, causing clinical confusion with malignant tumours. When seen in the clinic it is often misdiagnosed as a cyst, polyp or angioma. The diagnosis of MGLA is made after a detailed histopathologic examination and it is often misdiagnosed as a low grade adenocarcinoma as both share a tubulo-papillary/glandular predominant growth pattern. The clue to diagnosis is the architectural circumscription, nodular architecture, bland nuclei, and lack of cytologic atypia along with apical snouting. The myoepithelial cells are usually inconspicuous on $\mathrm{HE}$, however, IHC for p63 highlights the spindled myoepithelial nuclei rimming the tubules and glands of MGLA.

MLGA/HP is known to affect women in a wide age range ranging from 25-89 years. Rare cases of HP in males involving the perianal region have been reported. Although, MLGA/HP are benign tumors, there are rare cases of 
malignant transformation to in situ ductal carcinoma and invasive adenocarcinoma. ${ }^{4}$ Our case showed no features of malignancy.

\section{Conclusion}

We report a case of Mammary like Gland Adenoma with diffuse strong GCDFP and GATA-3 positivity. These are uncommon tumors which are liable to be mistaken as an adenocarcinoma. Furthermore, we support the term Mammary like Gland Adenoma which more accurately reflects the true histogenesis of these tumors. This is the first report of a Mammary like gland adenoma with GATA-3 expression from India.

\section{Conflict of interest: None. Disclaimer: Nil.}

\section{References}

1. Scurry J, van der Putte SC, Pyman J, Chetty N, Szabo R. Mammary-like gland adenoma of the vulva: Review of 46 cases. Pathology. 2009; 41(4): 372-8.
2. van der Putte R. Anogenital "sweat" glands: Histology and pathology of a gland that may mimic mammary glands. The American journal of dermatopathology. 1991; 13(6): 557-67.

3. Chou J, Provot S, Werb Z. GATA3 in development and cancer differentiation: Cells GATA have it! Journal of cellular physiology. 2010; 222(1): 42-9.

4. Shah SS, Adelson M, Mazur MT. Adenocarcinoma in situ arising in vulvar papillary hidradenoma: Report of 2 cases. International Journal of Gynecological Pathology. 2008; 27(3): 453-6.

\section{Deval Parekh ${ }^{1}$, Sunaina Wadhwa ${ }^{2}$, Asima Mukhopadhyay ${ }^{3}$, Angad Singh ${ }^{4}$}

${ }^{1}$ Department of Pathology, Tata Medical Center, Kolkata, India; ${ }^{2}$ Department of Gynaecologic Oncology, Tata Medical Center, Kolkata, India;

${ }^{3}$ Department of Gynaecologic Oncology, Tata Medical Center, Kolkata, India; ${ }^{4}$ Department of Pathology, Tata Medical Center, Kolkata, India. 